\title{
Sitzung vom 26. April 1909.
}

Vorsitzender: Hr. Otto N. Witt, Präsident.

Das Protokoll der letzten Sitzung wird genehmigt.

Der Vorsitzende teilt mit, daß

\section{Alfred Partheil,}

außerordentlicher Professor der pharmazeutischen Chemie und Direktor des Pharmazeutisch-chemischen Laboratoriums der Universität Königsberg, im Alter von 48 Jahren verschieden ist. Partheil hat sich durch eine Reihe von Arbeiten auf dem Gebiet der organischen und pharmazeutischen Chemie bekannt gemacht.

Die Versammelten erheben sich zur Ebrung des Verstorbenen von ihren Sitzen.

Darauf legt der Vorsitzende den nachstehenden, mit einer großen Anzahl von Unterschriften versehenen Aufruf des Comité international en l'honneur de Amedeo Avogadro" vor, das unter dem Patronat Sr. Majestät des Königs Victor Emanuel III. von Italien und unter dem Ehrenpräsidium von St. Cannizzaro steht:

"C'est en 1911 que s'accomplira le Centenaire de la publication die célebre Mémoire d'A medeo Avogadro sur la constitution moléculaire des gaz. Le loi d'Avogadro et les conséquences que lui-même et d'autres savants en ont déduites, sont devenues, il y a longtemps, le pirot des théories chimiques et elles s'enchainent avec d'importantes recherches physiques.

Le Comité, qui vient de se constituer sur l'initiative de l'Académie Royale des Sciences de Turin, ne croit pas pouvoir mieux honorer le grand chimiste et physicien, que par la publication en un volume de ses travaux les plus importants, ainsi que par l'érection à Turin - où il est né et où il enseignait - d'un monument digne de lui.

$A$ cet effet le Comité fait appel à tous les chimistes et physiciens. dans l'espoir quils voudront tous contribuer à ce tardif, mais bien mérité hommage à la mémoire de cet homme grand autant que modeste, à qui la Science est redevable de tant de lumière."

Berichte d. D. Chem. Gesellschaft. Jahrg. XXXXII. 
Der Vorsitzende spricht die Hofinung aus, daß dieses Unternehmen auch im Kreise der deutschen Chemiker lebhafte Unterstiitzung finclen möge. Zahlungen sind zu richten an deu Schatzmeister der "Académie des sciences, Turin, Via Maria Vitoria 3 . Auch ist die Geschäftsstelle der Deutschen Chemisclen Gesellschait, Berlin W. 10, Sigismundstrabe 4 bereit, Beiträge bis zum 1. Juni d. J. entgegenzunehmen :nd dem Komites zu whermitteln.

Als außerordentliche Mitglieder sind aufgenommen die HH'n.:

Kantscheti, W., Charlottenburg; Ballheimer, Tübingen;

Landsberger, F., $\quad$; Bindewald, M., 》;

Göckel, Jr. H., Berlin; Riedel, Dr. A., Halle;

Lecor, L., Paris;

Kansky, Berlin;

Wilke, lir. Ri., 》;

Lommel, V., Amani;

Stclzenberg, H., $》$ :

Baden, Dr. Ph., Luxemburg; Straul, F., Paria;

Ebert, Dr. G., Jeeds; Zanetti, L'rof. Dr. C. U., Bologna.

Kaltwasser, Dr. O., Dessau;

Als außerordentliche Mitglieder werden vorgeschlagen die HHrn.:

Ackermaun, Dr. Fritz, Chlandstr. 194, Charlottenburg (durch Otto N. Witt und $G$ von (iirsewald);

Delphin, Apotheker T., Upplaudsogatan 66, II, Stockholm (durch G. F. Bergh und A. Blom qaist);

Peski, A. J. jr. van, s'Gravendykwal $115 \mathrm{c}$, Nelit (durch I. Boeseken und H. ter Meulen);

Frimke, Werner, Breiteweg 228, II, Marburg,

Rosenthal, Leo, Schiitzenplatz 4,

Bernburg,

Huth. M a x E., Luisenstr. 2a, Halle a.S.,

(durch T). Vor-

länder und

K. 'T'ubandt);

Hanus, Prof. Josef, Technische Hocinschule, Prag II (durch G. Baborowsky und B. Kuzma);

Kutscheroff, Dr. Leo, Alexandrowski Prosp. 4, St. Petersburg (durch .1. Salkind und D. (iarulner);

Weller, Dr. Arnold, Kurfürstendamm 32, Berlin W. 15 (durch F. Sachs und P. Jacobson;;

Arndt, Dr. Fritz, Friedrichstr. 33, Freiburg i. B. (dureh I. (iattermann unil W. Meigen). 
Fïr die Bibliothek sind als Geschenke eingegangen:

538. Miller, W. v. und Kiliani, H. Kurzes Lehrbuch der analytischen Chemie. Bearbeitet von H. Kiliani. 6. Auflage. München 1909.

26. Fehling, H. v. Neues Handwörterbuch der Chemie, fortgesetzt von C. Hell und C. Hänßermann. Lieferung 107. Braunschweig 1909.

209. Bericht ron Schimmel \& Co. Miltitz, April 1909, nebst m. Irbeitsstätten der Firma Schimmel \& Co.«

1580. Gmelin-Krauts Hanclbuch der organischen Chemie, herausgegeben von C. Friedheim. 7. Aullage. 83-91. Lieferung. Heidellerg 1909.

Der Vorsitzende:

Otto N. Witt.
Der Schriftfiibrer:

i. V. F. Mylius.

\section{Mitteilungen.}

\section{J. v. Braun: Über die Chlorphosphor-Aufspaltung des Camphidins und einige neue Derivate des Benzoyl- $\varepsilon$-chlor- amylamins.}

[Aus dem Chemischen Institut der Lniversität Gütting'n]]

[Eingegangen am 3. April 1909.]

Nachdem es mir in den letzten Jahren an einer Reihe relativ einfach gebauter sekundärer, cyclischer Basen gelungen ist zu zeigen, daß ihre Aufspaltung mit Hilfe von Fünffachchlor- resp. Fünffachbromphosphor zu recht günstigen Resultaten fuihrt ${ }^{1}$ ), bin ich neuerdings dazu übergegangen, die Tersuche auch auf einige komplizierter gebaute, namentlich polycycliscle Basen (z. B. Campbidin, Nortropan, hydrierte Carbazole usw.) auszudehnen, und es hat sich dabei bisher heransgestellt, daß sich die Methode auch hier glatt anwenden läBt. Die Ringsprengung der Basen führt zu wobl charakterisierten Umwandlungsprodukten, die auf anderen Wegen aus ibnen nicht erbalten werden können. In der vorliegenden Mitteilung möchte ich kurz die Verhältnisse beim Camphidin schildern, da die bisherigen Resultate im gewissen Sinne als abgerundet angesehen werden können und die weitere Ausdebnung und Vervollständigung der Versucbe bei der Schwierigkeit, mit der die Beschaffung grïßerer Mengen des Canı-

1) Vurgi. diese Berichte 37, 2915, 45icl [1904]; 38, 179, 2336 [1905]; $39,4119,436.5[1906]$. 